\title{
Explicit iterations for Lipschitzian semigroups with the Meir-Keeler type contraction in Banach spaces
}

\author{
Siwaporn Saewan ${ }^{1 *}$ and Poom Kumam²
}

\section{"Correspondence:}

si_wa_pon@hotmail.com;

siwaporn@tsu.ac.th;

poom.kum@kmutt.ac.th

'Department of Mathematics and

Statistics, Faculty of Science, Thaksin

University (TSU), Phatthalung,

93110, Thailand

${ }^{2}$ Department of Mathematics, Faculty of Science, King Mongkut's University of Technology Thonburi (KMUTT), Bangkok, 10140, Thailand

\begin{abstract}
In this paper, we introduce and prove strong convergence theorems for a new viscosity iteration scheme for approximating common fixed points of a Lipschitzian semigroup on a compact and convex subset of a smooth Banach space. Our results extend and improve recent results.

MSC: $47 \mathrm{H} 09 ; 47 \mathrm{H} 10$

Keywords: Meir-Keeler type mapping; Lipschitzian mapping; variational inequality; viscosity approximation method; fixed point
\end{abstract}

\section{Introduction}

Let $C$ be a nonempty, closed and convex subset of a Banach space $E$. Recall that a self mapping $f: E \rightarrow E$ is an $\alpha$-contraction on $C$ if there exists a constant $\alpha \in(0,1)$ such that for any $x, y \in E$, we have

$$
\|f(x)-f(y)\| \leq \alpha\|x-y\|
$$

A function $\psi: \mathbb{R}^{+} \rightarrow \mathbb{R}^{+}$is said to be an $L$-function if $\psi(0)=0, \psi(t)>0$ for any $t>0$, and for every $t>0$ and $s>0$, there exists $u>s$ such that $\psi(t) \leq s$ for all $t \in[s, u]$. This implies that $\psi(t)<t$ for all $t>0$.

A mapping $f: E \rightarrow E$ is said to be a $(\psi, L)$-contraction if there exists an $L$-function $\psi$ : $\mathbb{R}^{+} \rightarrow \mathbb{R}^{+}$such that

$$
\|f(x)-f(y)\| \leq \psi\|x-y\|, \quad \forall x, y \in E \text { with } x \neq y .
$$

If $\psi(t)=k t$ for all $t>0$, where $k \in(0,1)$, then $f$ is a contraction.

A mapping $f$ is called a Meir-Keeler type mapping if for each $\epsilon>0$, there exists $\delta(\epsilon)>0$ such that for all $x, y \in E$, if $\epsilon<\|x-y\|<\epsilon+\delta$, then $\|f(x)-f(y)\|<\epsilon$.

A mapping $T: C \rightarrow C$ is said to be

(i) nonexpansive if

$$
\|T x-T y\| \leq\|x-y\|, \quad \forall x, y \in C
$$

\section{Springer}

(c) 2012 Saewan and Kumam; licensee Springer. This is an Open Access article distributed under the terms of the Creative Commons Attribution License (http://creativecommons.org/licenses/by/2.0), which permits unrestricted use, distribution, and reproduction in any medium, provided the original work is properly cited. 
(ii) Lipschitzian with a Lipschitz constant $l>0$ if

$$
\|T x-T y\| \leq l\|x-y\|, \quad \forall x, y \in C ;
$$

(iii) asymptotically nonexpansive if there exists a sequence $\left\{k_{n}\right\}$ of positive numbers satisfying the property $\lim _{n \rightarrow \infty} k_{n}=1$ and

$$
\left\|T^{n} x-T^{n} y\right\| \leq k_{n}\|x-y\|, \quad \forall x, y \in C
$$

Every nonexpansive mappings are asymptotically nonexpansive with respect to the sequence $k_{n}=1, n \in \mathbb{N}$. Also, every asymptotically nonexpansive mappings are uniformly $l$-Lipschitzian with $l=\sup _{n \in \mathbb{N}} k_{n}$.

Let $S$ be a semigroup. Then a family $\mathcal{S}=\left\{T_{s}: s \in S\right\}$ of mappings of $C$ into itself is called Lipschitzian mappings on $C$ if for each $s \in S$, the mapping $T_{s}$ is a Lipschitzian mapping on $C$ with a Lipschitz constant $k_{s}$, and $T_{s t}=T_{s} T_{t}$ for all $s, t \in S$. A family $\mathcal{S}$ is called a Lipschitzian semigroup on $C$ if it satisfies the following:

1. $T_{s t} x=T_{s} T_{t} x$ for all $s, t \in S$ and $x \in C$;

2. for each $s \in S, T_{s}$ is a Lipschitzian mapping of $C$ into itself, i.e., there is $k_{s} \geq 0$ such that

$$
\left\|T_{s} x-T_{s} y\right\| \leq k_{s}\|x-y\| \quad \text { for all } x, y \in C .
$$

A Lipschitzian semigroup $\mathcal{S}$ is called uniformly l-Lipschitzian if $k_{s}=l$ for all $s \in S$.

Let $F(\mathcal{S})$ denote the common fixed point set $\left\{x \in C: T_{s} x=x, \forall s \in S\right\}$ of the mappings $T_{s}$. For a semigroup $S$, we define a partial preordering $\prec$ on $S$ by $a \prec b$ if and only if $a S \supset b S$. If $S$ is a left reversible semigroup (i.e., $a S \cap b S \neq \emptyset$ for $a, b \in S$ ), then it is a directed set. (Indeed, for every $a, b \in S$, applying $a S \cap b S \neq \emptyset$, there exist $a^{\prime}, b^{\prime} \in S$ with $a a^{\prime}=b b^{\prime}$; by taking $c=a a^{\prime}=b b^{\prime}$, we have $c S \subseteq a S \cap b S$, and then $a \prec c$ and $b \prec c$.)

Let $\mathcal{S}=\left\{T_{s}: s \in S\right\}$ be a representation of a left reversible semigroup $S$ as Lipschitzian mappings on $C$ with Lipschitz constants $\left\{k_{s}: s \in S\right\}$. We shall say that $\mathcal{S}$ is an asymptotically nonexpansive semigroup on $C$, if there holds the uniform Lipschitzian condition $\lim _{s} k_{s} \leq 1$ on the Lipschitz constants.

In 1953, Mann [1] introduced an iterative method as follows: a sequence $\left\{x_{n}\right\}$ defined by

$$
x_{n+1}=\alpha_{n} x_{n}+\left(1-\alpha_{n}\right) T x_{n},
$$

where the initial guess element $x_{0} \in C$ is arbitrary and $\left\{\alpha_{n}\right\}$ is a sequence of real numbers in $[0,1]$. The Mann iteration can guarantee in general only weak convergence. The Mann iteration has been extensively investigated for nonexpansive mappings and modified for strong convergence. Later, in 2000, Reich and Zaslavski [2] introduced the KrasnoselskiiMann iterations of a generic nonexpansive operator on a closed and convex, but not necessarily bounded, subset of a hyperbolic space with a unique fixed point.

In 1967, Halpern [3] considered the following algorithm:

$$
x_{n+1}=\alpha_{n} x+\left(1-\alpha_{n}\right) T x_{n},
$$

where $T$ is nonexpansive and the initial guess element $x \in C$ is arbitrary. 
In 2004, Xu [4] introduced and proved the following viscosity approximation methods for nonexpansive mappings in a uniformly smooth Banach space:

$$
x_{n+1}=\alpha_{n} f\left(x_{n}\right)+\left(1-\alpha_{n}\right) T x_{n},
$$

where $f: C \rightarrow C$ is a contraction mapping.

In 2007, Lau, Miyake and Takahashi [5] introduced the following Mann's implicit iteration process:

$$
x_{n+1}=\alpha_{n} x+\left(1-\alpha_{n}\right) T\left(\mu_{n}\right) x_{n},
$$

for a semigroup $\mathcal{S}=\left\{T_{s}: s \in S\right\}$ of nonexpansive mappings on a compact and convex subset $C$ of a smooth and strictly convex Banach space.

In the same year, Zhang et al. [6] introduced the following composite iteration scheme:

$$
\left\{\begin{array}{l}
y_{n}=\beta_{n} x_{n}+\left(1-\beta_{n}\right) T\left(t_{n}\right) x_{n}, \\
x_{n+1}=\alpha_{n} x+\left(1-\alpha_{n}\right) y_{n},
\end{array}\right.
$$

where $\{T(t): t \geq 0\}$ is a nonexpansive semigroup, $x$ is an arbitrary point in $C$. Under a suitable condition, they proved strong convergence theorems of an explicit composite iteration scheme for nonexpansive semigroups in a reflexive Banach space with a uniformly Gâteaux differentiable norm, uniformly smooth Banach space and uniformly convex Banach space with a weakly continuous normalized duality mapping.

In 2008, Shahram Saeidi [7] introduced the following viscosity iterative scheme:

$$
x_{n+1}=\alpha_{n} f\left(x_{n}\right)+\beta_{n} x_{n}+\gamma_{n} T\left(\mu_{n}\right) x_{n}
$$

for a representation of $S$ as Lipschitzian mappings on a compact and convex subset $C$ of a smooth Banach space $E$ with respect to a left regular sequence $\left\{\mu_{n}\right\}$ of means defined on an appropriate invariant subspace of $l^{\infty}(S)$; for some related results, we refer the readers to $[8,9]$.

Motivated and inspired by the idea of Zhang et al. [6] and Saeidi [7], we introduce the explicit viscosity iterative process by Meir-Keeler type contraction in a smooth Banach space. Then we prove that the sequence $\left\{x_{n}\right\}$ converges strongly to a common fixed point of $\mathcal{S}=\left\{T_{s}: s \in S\right\}$, where $S$ is a left reversible semigroup, which is the unique solution of the variational inequality

$$
\langle(f-I) q, J(p-q)\rangle \leq 0, \quad \forall p \in F(\mathcal{S})
$$

\section{Preliminaries}

Let $E$ be a Banach space and let $E^{\prime \prime}$ be the topological dual of $E$. The value of $x^{\prime \prime} \in E^{\prime \prime}$ at $x \in E$ will be denoted by $\left\langle x, x^{*}\right\rangle$ or $x^{*}(x)$. With each $x \in E$, we associate the set

$$
J(x)=\left\{x^{*} \in E^{*}:\left\langle x, x^{*}\right\rangle=\left\|x^{*}\right\|^{2}=\|x\|^{2}\right\} .
$$

Using the Hahn-Banach theorem, it immediately follows that $J(x) \neq \emptyset$ for each $x \in E$. A Banach space $E$ is said to be smooth if the duality mapping $J$ of $E$ is single-valued. We 
know that if $E$ is smooth, then $J$ is norm-to-weak" continuous; see [8, 9]. Let $E$ be a Banach space and let $C$ be a closed and convex subset of $E$. Then

$$
\|x-y\|^{2}=\|x\|^{2}-\|y\|^{2}-2\langle x-y, y\rangle
$$

and

$$
\|\lambda x+(1-\lambda) y\|^{2}=\lambda\|x\|^{2}+(1-\lambda)\|y\|^{2}-\lambda(1-\lambda)\|x-y\|^{2},
$$

for all $x, y \in E$ and $\lambda \in[0,1]$.

Let $S$ be a semigroup. We denote by $l^{\infty}(S)$ the Banach space of all bounded real valued functions on $S$ with a supremum norm. For each $s \in S$, we define $l_{s}$ and $r_{s}$ on $l^{\infty}(S)$ by $\left(l_{s} g\right)(t)=g(s t)$ and $\left(r_{s} g\right)(t)=g(t s)$ for each $t \in S$ and $g \in l^{\infty}(S)$. Let $X$ be a subspace of $l^{\infty}(S)$ containing 1 and let $X^{*}$ be its topological dual. An element $\mu$ of $X^{*}$ is said to be a mean on $X$ if $\|\mu\|=\mu(1)=1$. We often write $\mu_{t}(g(t))$ instead of $\mu(g)$ for $\mu \in X^{*}$ and $g \in X$. Let $X$ be left invariant (resp. right invariant), i.e., $l_{s}(X) \subset X$ (resp. $\left.r_{s}(X) \subset X\right)$ for each $s \in S$. A mean $\mu$ on $X$ is said to be left invariant (resp. right invariant) if $\mu\left(l_{s} g\right)=\mu(g)$ (resp. $\left.\mu\left(r_{s} g\right)=\mu(g)\right)$ for each $s \in S$ and $g \in X$. A subspace $X$ is said to be left (resp. right) amenable if $X$ has a left (resp. right) invariant mean. A semigroup $X$ is amenable if $X$ is both left and right amenable. If a semigroup $S$ is left amenable, then $S$ is left reversible [10, 11].

A net $\left\{\mu_{\alpha}\right\}$ of means on $X$ is said to be strongly left regular if

$$
\lim _{\alpha}\left\|l_{s}^{*} \mu_{\alpha}-\mu_{\alpha}\right\|=0
$$

for each $s \in S$, where $l_{s}^{*}$ is the adjoint operator of $l_{s}$. Let $C$ be a nonempty, closed and convex subset of $E$. Throughout this paper, $S$ will always denote a semigroup with an identity $e . S$ is called left reversible if any two right ideals in $S$ have nonvoid intersection, i.e., $a S \cap b S \neq \emptyset$ for any $a, b \in S$. In this case, we can define a partial ordering $\prec$ on $S$ by $a \prec b$ if and only if $a S \supset b S$. It is easy to see $t \prec t s$ (for all $t, s \in S$ ). Further, if $t \prec s$, then $p t \prec p s$ for all $p \in S$. If a semigroup $S$ is left amenable, then $S$ is left reversible. But the converse is false. Denote by $C_{a}$ the set of almost periodic elements in $C$, i.e., all $x \in C$ such that $\mathcal{S}=\left\{T_{s} x: s \in S\right\}$ is relatively compact in the norm topology of $E$. We will call a subspace $X$ of $l^{\infty}(S), \mathcal{S}$-stable if the functions $s \mapsto\left\langle T_{s} x, x^{*}\right\rangle$ and $s \mapsto\left\|T_{s} x-y\right\|$ on $S$ are in $X$ for all $x, y \in C$ and $x^{*} \in E^{*}$. We know that if $\mu$ is a mean on $X$ and if for each $x^{*} \in E^{*}$, the function $s \mapsto\left\langle T_{s} x, x^{*}\right\rangle$ is contained in $X$ and $C$ is weakly compact, then there exists a unique point $x_{0}$ of $E$ such that

$$
\mu_{s}\left\langle T_{s} x, x^{*}\right\rangle=\left\langle x_{0}, x^{*}\right\rangle
$$

for each $x^{*} \in E^{*}$. We denote such a point $x_{0}$ by $T(\mu) x$. Note that $T(\mu) z=z$ for each $z \in F(\mathcal{S})$; see [12-14]. Let $D$ be a subset of $B$ where $B$ is a subset of a Banach space $E$ and let $P$ be a retraction of $B$ onto $D$. Then $P$ is said to be sunny [15] if for each $x \in B$ and $t \geq 0$ with $P x+t(x-P x) \in B$,

$$
P(P x+t(x-P x))=P x .
$$

A subset $D$ is said to be a sunny nonexpansive retract of $B$ if there exists a sunny nonexpansive retraction $P$ of $B$ onto $D$. If $E$ is smooth and $P$ is a retraction of $B$ onto $D$, then $P$ 
is sunny and nonexpansive if and only if for each $x \in B$ and $z \in D$,

$$
\langle x-P x, J(z-P x)\rangle \leq 0
$$

For more details, see $[8,9]$.

Lemma 2.1 ([16]) Let $S$ be a left reversible semigroup and let $\mathcal{S}=\left\{T_{s}: s \in S\right\}$ be a representation of $S$ as Lipschitzian mappings from a nonempty, weakly compact and convex subset $C$ of a Banach space $E$ into $C$, with the uniform Lipschitzian condition $\lim _{s} k_{s} \leq 1$ on the Lipschitz constants of the mappings. Let $X$ be a left invariant $\mathcal{S}$-stable subspace of $l^{\infty}(S)$ containing 1 , and $\mu$ be a left invariant mean on $X$. Then $F(\mathcal{S})=F(T(\mu)) \cap C_{a}$.

Corollary 2.2 ([7]) Let $\left\{\mu_{n}\right\}$ be an asymptotically left invariant sequence of means on $X$. If $z \in C_{a}$ and $\liminf _{n \rightarrow \infty}\left\|T\left(\mu_{n}\right) z-z\right\|=0$, then $z$ is a common fixed point of $\mathcal{S}$.

Lemma 2.3 ([7]) Let $S$ be a left reversible semigroup and let $\mathcal{S}=\left\{T_{s}: s \in S\right\}$ be a representation of $S$ as Lipschitzian mappings from a nonempty weakly compact and convex subset $C$ of a Banach space $E$ into $C$, with the uniform Lipschitzian condition $\lim _{s} k_{s} \leq 1$ on the Lipschitz constants of the mappings. Let $X$ be a left invariant subspace of $l^{\infty}(S)$ containing 1 such that the mappings $s \mapsto\left\langle T_{s} x, x^{*}\right\rangle$ be in $X$ for all $x \in X$ and $x^{*} \in E^{*}$, and $\left\{\mu_{n}\right\}$ be a strongly left regular sequence of means on $X$. Then

$$
\limsup _{n \rightarrow \infty} \sup _{x, y \in C}\left(\left\|T\left(\mu_{n}\right) x-T\left(\mu_{n}\right) y\right\|-\|x-y\|\right) \leq 0 .
$$

Remark 2.4 From Lemma 2.3, taking

$$
c_{n}=\sup _{x, y \in C}\left(\left\|T\left(\mu_{n}\right) x-T\left(\mu_{n}\right) y\right\|-\|x-y\|\right), \quad \forall n,
$$

we obtain that $\lim _{\sup _{n \rightarrow \infty}} c_{n} \leq 0$. Moreover,

$$
\left\|T\left(\mu_{n}\right) x-T\left(\mu_{n}\right) y\right\| \leq\|x-y\|+c_{n}, \quad \forall x, y \in C .
$$

Corollary 2.5 ([7]) Let $S$ be a left reversible semigroup and let $\mathcal{S}=\left\{T_{s}: s \in S\right\}$ be a representation of $S$ as Lipschitzian mappings from a nonempty, compact and convex subset $C$ of a Banach space $E$ into $C$, with the uniform Lipschitzian condition $\lim _{s} k_{s} \leq 1$. Let X be a left invariant $\mathcal{S}$-stable subspace of $l^{\infty}(S)$ containing 1 , and $\mu$ be a left invariant mean on $X$. Then $T(\mu)$ is nonexpansive and $F(\mathcal{S}) \neq \emptyset$. Moreover, if $E$ is smooth, then $F(\mathcal{S})$ is a sunny nonexpansive retract of $C$ and the sunny nonexpansive retraction of $C$ onto $F(\mathcal{S})$ is unique.

Lemma 2.6 ([8,9]) Let E be a real Banach space. Then, for any given $x, y \in E$ and $j(x+y) \in$ $J(x+y)$, the following inequality holds:

$$
\|x+y\|^{2} \leq\|x\|^{2}+2\langle y, j(x+y)\rangle
$$

Lemma 2.7 ([17]) Let $\left\{x_{n}\right\}$ and $\left\{y_{n}\right\}$ be two bounded sequences in a Banach space E and let $\left\{\beta_{n}\right\}$ be a sequence in $[0,1]$ with $0<\liminf _{n \rightarrow \infty} \beta_{n} \leq \limsup _{n \rightarrow \infty} \beta_{n}<1$. Suppose that 
$x_{n+1}=\left(1-\beta_{n}\right) y_{n}+\beta_{n} x_{n}$ for all integers $n \geq 0$ and $\limsup _{n \rightarrow \infty}\left(\left\|y_{n+1}-y_{n}\right\|-\left\|x_{n+1}-x_{n}\right\|\right) \leq 0$.

Then $\lim _{n \rightarrow \infty}\left\|y_{n}-x_{n}\right\|=0$.

Lemma 2.8 ([4]) Assume that $\left\{a_{n}\right\}$ is a sequence of nonnegative real numbers such that

$$
a_{n+1} \leq\left(1-\sigma_{n}\right) a_{n}+\rho_{n}
$$

where $\left\{\alpha_{n}\right\}$ is a sequence in $(0,1)$ and $\left\{\delta_{n}\right\}$ is a sequence in $\mathbb{R}$ such that

(1) $\sum_{n=1}^{\infty} \alpha_{n}=\infty$;

(2) $\lim \sup _{n \rightarrow \infty} \frac{\delta_{n}}{\alpha_{n}} \leq 0$ or $\sum_{n=1}^{\infty}\left|\delta_{n}\right|<\infty$.

Then $\lim _{n \rightarrow \infty} a_{n}=0$.

Lemma 2.9 ([18]) Let $(Y, d)$ be a metric space and let $f: Y \rightarrow Y$ be a mapping. The following assertions are equivalent:

1. $f$ is a Meir-Keeler type mapping;

2. there exists a L-function $\psi: \mathbb{R}^{+} \rightarrow \mathbb{R}^{+}$such that $f$ is a $(\psi, L)$-contraction.

Lemma 2.10 ([19]) Let E be a Banach space and let $C$ be a convex subset of E. Let $T: C \rightarrow$ $C$ be a nonexpansive mapping and $f$ be $a(\psi, L)$-contraction. Then the following assertions hold:

1. $T \circ f$ is a $(\psi, L)$-contraction on $C$ and has a unique fixed point in $C$;

2. for each $\alpha \in(0,1)$, the mapping $x \rightarrow \alpha f(x)+(1-\alpha)$ Tx is of Meir-Keeler-type and it has a unique fixed point in $C$.

Lemma 2.11 ([19]) Let E be a Banach space and let $C$ be a convex subset of E. Let $f: C \rightarrow C$ be a Meir-Keeler-type contraction. Then for each $\epsilon>0$, there exists $r \in(0,1)$ such that, for each $x, y \in C$ with $\|x-y\| \geq \epsilon$, we have $\|f(x)-f(y)\| \leq r\|x-y\|$.

\section{Main results}

In this paper, we suppose that $\psi$ from the definition of $(\psi, L)$-contraction is continuous, strictly increasing. Let $\eta(t)=t-\psi(t)$ for all $t \in \mathbb{R}^{+}$, we have that $\lim _{t \rightarrow \infty} \eta(t)=\infty$ and $\eta(t)$ is strictly increasing and onto. Consequently, we have that and $\eta(t)$ is a bijection on $\mathbb{R}^{+}$.

Theorem 3.1 Let $C$ be a nonempty, compact and convex subset of a smooth Banach space $E$. Let $S$ be a left reversible semigroup and $\mathcal{S}=\left\{T_{s}: s \in S\right\}$ be a representation of $S$ as Lipschitzian mappings from $C$ into itself, with the uniform Lipschitzian condition $\lim _{s} k_{s} \leq 1$, and $f$ be a Meir-Keeler contraction of $C$ into itself. Let $X$ be a left invariant $\mathcal{S}$-stable subspace of $l^{\infty}(S)$ containing $1,\left\{\mu_{n}\right\}$ be a strongly left regular sequence of means on $X$ such that $\lim _{n \rightarrow \infty}\left\|\mu_{n+1}-\mu_{n}\right\|=0$ and $\left\{c_{n}\right\}$ be the sequence defined in (2.4) with $\limsup _{n \rightarrow \infty} c_{n} \leq 0$. Suppose the sequences $\left\{\alpha_{n}\right\},\left\{\beta_{n}\right\},\left\{\gamma_{n}\right\}$ and $\left\{\delta_{n}\right\}$ in $(0,1)$ satisfy $\alpha_{n}+\beta_{n}+\gamma_{n}=1, n \geq 1$. The following conditions are satisfied:

(i) $\lim _{n \rightarrow \infty} \alpha_{n}=0$ and $\sum_{n=0}^{\infty} \alpha_{n}=\infty$;

(ii) $\lim _{n \rightarrow \infty} \delta_{n}=0$;

(iii) $\lim \sup _{n \rightarrow \infty} \frac{c_{n}}{\alpha_{n}} \leq 0$;

(iv) $0<\liminf _{n \rightarrow \infty} \beta_{n} \leq \limsup _{n \rightarrow \infty} \beta_{n}<1$. 
For arbitrary $x_{1} \in C$, generate a sequence $\left\{x_{n}\right\}$ by

$$
\left\{\begin{array}{l}
y_{n}=\delta_{n} x_{n}+\left(1-\delta_{n}\right) T\left(\mu_{n}\right) x_{n}, \\
x_{n+1}=\alpha_{n} f\left(x_{n}\right)+\beta_{n} x_{n}+\gamma_{n} y_{n} .
\end{array}\right.
$$

Then $\left\{x_{n}\right\}$ converges strongly to $q \in F(\mathcal{S})$, which is the unique solution of the variational inequality

$$
\langle(f-I) q, J(p-q)\rangle \leq 0, \quad \forall p \in F(\mathcal{S})
$$

Equivalently, we have $q=P f q$, where $P$ is the unique sunny nonexpansive retraction of $C$ onto $F(\mathcal{S})$.

Proof First, we prove that $\left\{x_{n}\right\}$ is bounded. That is, if we take a point $p \in F(\mathcal{S})$, we will show that $\left\|x_{n}-p\right\| \leq M$ for all $n \in \mathbb{N}$. Let $p \in F(\mathcal{S})$. It is obvious that $M=\max \left\{\left\|x_{1}-p\right\|, \eta^{-1} \| f(p)-\right.$ $p \|\}$. By induction we suppose that $M=\max \left\{\left\|x_{k}-p\right\|, \eta^{-1}\|f(p)-p\|\right\}$, where $k \in \mathbb{N}$. Since $T\left(\mu_{k}\right)$ is nonexpansive, we have

$$
\begin{aligned}
\left\|y_{k}-p\right\| & =\left\|\delta_{n} x_{k}+\left(1-\delta_{k}\right) T\left(\mu_{k}\right) x_{k}-p\right\| \\
& \leq \delta_{k}\left\|x_{k}-p\right\|+\left(1-\delta_{k}\right)\left\|T\left(\mu_{k}\right) x_{k}-p\right\| \\
& \leq \delta_{k}\left\|x_{k}-p\right\|+\left(1-\delta_{k}\right)\left(\left\|x_{k}-p\right\|\right) \\
& \leq\left\|x_{k}-p\right\| .
\end{aligned}
$$

Since $f$ is a Meir-Keeler contraction, we have that

$$
\begin{aligned}
\left\|x_{k+1}-p\right\| & =\left\|\alpha_{k} f\left(x_{k}\right)+\beta_{k} x_{k}+\gamma_{k} y_{k}-p\right\| \\
& =\left\|\alpha_{k}\left(f\left(x_{k}\right)-p\right)+\beta_{k}\left(x_{k}-p\right)+\gamma_{n}\left(y_{k}-p\right)\right\| \\
& \leq \alpha_{k}\left\|f\left(x_{k}\right)-p\right\|+\beta_{k}\left\|x_{k}-p\right\|+\gamma_{k}\left\|y_{k}-p\right\| \\
& \leq \alpha_{k}\left\|f\left(x_{k}\right)-f(p)\right\|+\alpha_{k}\|f(p)-p\|+\beta_{k}\left\|x_{k}-p\right\|+\gamma_{k}\left\|y_{k}-p\right\| \\
& \leq \alpha_{k} \psi\left\|x_{k}-p\right\|+\alpha_{k}\|f(p)-p\|+\beta_{k}\left\|x_{k}-p\right\|+\gamma_{k}\left\|y_{k}-p\right\| \\
& =\alpha_{k} \psi\left\|x_{k}-p\right\|+\left(\beta_{k}+\gamma_{k}\right)\left\|x_{k}-p\right\|+\alpha_{k} \eta\left(\eta^{-1}\right)\|f(p)-p\| \\
& =\alpha_{k} \psi\left\|x_{k}-p\right\|+\left(1-\alpha_{k}\right)\left\|x_{k}-p\right\|+\alpha_{k} \eta\left(\eta^{-1}\right)\|f(p)-p\| \\
& \leq \alpha_{k} \psi(M)+\left(1-\alpha_{k}\right)(M)+\alpha_{k} \eta(M) \\
& \leq \alpha_{k} \psi(M)+\left(1-\alpha_{k}\right)(M)+\alpha_{k}(M-\psi(M)) \\
& =M .
\end{aligned}
$$

That is, $\left\{x_{k+1}\right\}$ is bounded. By induction we have that $\left\{x_{n}\right\}$ is bounded, and so are the sequences $\left\{f\left(x_{n}\right)\right\},\left\{T\left(\mu_{n}\right) x_{n}\right\}$ and $\left\{y_{n}\right\}$. As $T\left(\mu_{n}\right)$ is bounded, we have $\left\{T_{s} x_{n}\right\}$ is also bounded. Denote $D=\sup _{s \in S}\left\|T_{s} x_{n}\right\|$, then it follows that

$$
\begin{aligned}
\left\|T\left(\mu_{n+1}\right) x_{n}-T\left(\mu_{n}\right) x_{n}\right\| & =\sup \left\{\left|\left\langle T\left(\mu_{n+1}\right) x_{n}-T\left(\mu_{n}\right) x_{n}, z^{*}\right\rangle\right|: z^{*} \in E^{*},\left\|z^{*}\right\|=1\right\} \\
& =\sup \left\{\left|\left(\mu_{n+1}\right)_{s}\left\langle T_{s} x_{n}, z^{*}\right\rangle-\left(\mu_{n}\right)_{s}\left\langle T_{s} x_{n}, z^{*}\right\rangle\right|: z^{*} \in E^{*},\left\|z^{*}\right\|=1\right\}
\end{aligned}
$$




$$
\begin{aligned}
& \leq\left\|\mu_{n+1}-\mu_{n}\right\| \sup _{s \in S}\left\|T_{s} x_{n}\right\|\left\|z^{*}\right\| \\
& \leq\left\|\mu_{n+1}-\mu_{n}\right\| \sup _{s \in S}\left\|T_{s} x_{n}\right\| \\
& =\left\|\mu_{n+1}-\mu_{n}\right\| D .
\end{aligned}
$$

Since $\lim _{n \rightarrow \infty}\left\|\mu_{n+1}-\mu_{n}\right\|=0$, we obtain that

$$
\lim _{n \rightarrow \infty}\left\|T\left(\mu_{n+1}\right) x_{n}-T\left(\mu_{n}\right) x_{n}\right\|=0 .
$$

Next, we will show that $\lim _{n \rightarrow \infty}\left\|x_{n+1}-x_{n}\right\|=0$ and by Lemma 2.3, we observe that

$$
\begin{aligned}
\left\|y_{n+1}-y_{n}\right\|= & \left\|\delta_{n+1} x_{n+1}+\left(1-\delta_{n+1}\right) T\left(\mu_{n+1}\right) x_{n+1}-\left(\delta_{n} x_{n}+\left(1-\delta_{n}\right) T\left(\mu_{n}\right) x_{n}\right)\right\| \\
= & \| \delta_{n+1} x_{n+1}-\delta_{n+1} x_{n}+\delta_{n+1} x_{n}+\left(1-\delta_{n+1}\right) T\left(\mu_{n+1}\right) x_{n+1} \\
& -\left(1-\delta_{n+1}\right) T\left(\mu_{n}\right) x_{n}+\left(1-\delta_{n+1}\right) T\left(\mu_{n}\right) x_{n}-\delta_{n} x_{n}-\left(1-\delta_{n}\right) T\left(\mu_{n}\right) x_{n} \| \\
= & \| \delta_{n+1}\left(x_{n+1}-x_{n}\right)+\left(\delta_{n+1}-\delta_{n}\right) x_{n}+\left(1-\delta_{n+1}\right)\left(T\left(\mu_{n+1}\right) x_{n+1}\right. \\
& \left.-T\left(\mu_{n}\right) x_{n}\right)+\left(\delta_{n}-\delta_{n+1}\right) T\left(\mu_{n}\right) x_{n} \| \\
\leq & \delta_{n+1}\left\|x_{n+1}-x_{n}\right\|+\left|\delta_{n+1}-\delta_{n}\right|\left(\left\|x_{n}\right\|+\left\|T\left(\mu_{n}\right) x_{n}\right\|\right) \\
& +\left\|T\left(\mu_{n+1}\right) x_{n+1}-T\left(\mu_{n}\right) x_{n}\right\| \\
\leq & \delta_{n+1}\left\|x_{n+1}-x_{n}\right\|+\left|\delta_{n+1}-\delta_{n}\right|\left(\left\|x_{n}\right\|+\left\|T\left(\mu_{n}\right) x_{n}\right\|\right) \\
& +\left\|T\left(\mu_{n+1}\right) x_{n+1}-T\left(\mu_{n}\right) x_{n+1}\right\|+\left\|T\left(\mu_{n}\right) x_{n+1}-T\left(\mu_{n}\right) x_{n}\right\| \\
\leq & \delta_{n+1}\left\|x_{n+1}-x_{n}\right\|+\left|\delta_{n+1}-\delta_{n}\right|\left(\left\|x_{n}\right\|+\left\|T\left(\mu_{n}\right) x_{n}\right\|\right) \\
& +\left\|T\left(\mu_{n+1}\right) x_{n+1}-T\left(\mu_{n}\right) x_{n+1}\right\|+\left\|x_{n+1}-x_{n}\right\|+c_{n} .
\end{aligned}
$$

Setting $x_{n+1}=\left(1-\beta_{n}\right) z_{n}+\beta_{n} x_{n}$, we see that

$$
z_{n}=\frac{x_{n+1}-\beta_{n} x_{n}}{1-\beta_{n}}
$$

Then we compute

$$
\begin{aligned}
&\left\|z_{n+1}-z_{n}\right\| \\
&=\left\|\frac{x_{n+2}-\beta_{n+1} x_{n+1}}{1-\beta_{n+1}}-\frac{x_{n+1}-\beta_{n} x_{n}}{1-\beta_{n}}\right\| \\
&=\left\|\frac{\alpha_{n+1} f\left(x_{n+1}\right)+\gamma_{n+1} y_{n+1}}{1-\beta_{n+1}}-\frac{\alpha_{n} f\left(x_{n}\right)+\gamma_{n} y_{n}}{1-\beta_{n}}\right\| \\
&=\| \frac{\alpha_{n+1} f\left(x_{n+1}\right)+\gamma_{n+1} y_{n+1}}{1-\beta_{n+1}}-\frac{\alpha_{n+1} f\left(x_{n}\right)}{1-\beta_{n+1}}+\frac{\alpha_{n+1} f\left(x_{n}\right)}{1-\beta_{n+1}} \\
&=-\frac{\gamma_{n+1} y_{n}}{1-\beta_{n+1}}+\frac{\gamma_{n+1} y_{n}}{1-\beta_{n+1}}-\frac{\alpha_{n} f\left(x_{n}\right)+\gamma_{n} y_{n}}{1-\beta_{n}} \| \\
&= \| \frac{\alpha_{n+1}}{1-\beta_{n+1}}\left(f\left(x_{n+1}\right)-f\left(x_{n}\right)\right)+\frac{\gamma_{n+1}}{1-\beta_{n+1}}\left(y_{n+1}-y_{n}\right)
\end{aligned}
$$


Sewan and Kumam Journal of Inequalities and Applications 2012, 2012:279

Page 9 of 15

http://www.journalofinequalitiesandapplications.com/content/2012/1/279

$$
\begin{aligned}
& +\left(\frac{\alpha_{n+1}}{1-\beta_{n+1}}-\frac{\alpha_{n}}{1-\beta_{n}}\right) f\left(x_{n}\right)+\left(\frac{\gamma_{n+1}}{1-\beta_{n+1}}-\frac{\gamma_{n}}{1-\beta_{n}}\right) y_{n} \| \\
& =\| \frac{\alpha_{n+1}}{1-\beta_{n+1}}\left(f\left(x_{n+1}\right)-f\left(x_{n}\right)\right)+\frac{\gamma_{n+1}}{1-\beta_{n+1}}\left(y_{n+1}-y_{n}\right) \\
& +\left(\frac{\alpha_{n+1}}{1-\beta_{n+1}}-\frac{\alpha_{n}}{1-\beta_{n}}\right) f\left(x_{n}\right)+\left(\frac{1-\beta_{n+1}-\alpha_{n+1}}{1-\beta_{n+1}}-\frac{1-\beta_{n}-\alpha_{n}}{1-\beta_{n}}\right) y_{n} \| \\
& =\| \frac{\alpha_{n+1}}{1-\beta_{n+1}}\left(f\left(x_{n+1}\right)-f\left(x_{n}\right)\right)+\frac{\gamma_{n+1}}{1-\beta_{n+1}}\left(y_{n+1}-y_{n}\right) \\
& +\left(\frac{\alpha_{n+1}}{1-\beta_{n+1}}-\frac{\alpha_{n}}{1-\beta_{n}}\right) f\left(x_{n}\right)+\left(\frac{-\alpha_{n+1}}{1-\beta_{n+1}}+\frac{\alpha_{n}}{1-\beta_{n}}\right) y_{n} \| \\
& =\| \frac{\alpha_{n+1}}{1-\beta_{n+1}}\left(f\left(x_{n+1}\right)-f\left(x_{n}\right)\right)+\frac{\gamma_{n+1}}{1-\beta_{n+1}}\left(y_{n+1}-y_{n}\right) \\
& +\left(\frac{\alpha_{n+1}}{1-\beta_{n+1}}-\frac{\alpha_{n}}{1-\beta_{n}}\right) f\left(x_{n}\right)-\left(\frac{\alpha_{n+1}}{1-\beta_{n+1}}-\frac{\alpha_{n}}{1-\beta_{n}}\right) y_{n} \| \\
& =\| \frac{\alpha_{n+1}}{1-\beta_{n+1}}\left(f\left(x_{n+1}\right)-f\left(x_{n}\right)\right)+\frac{\gamma_{n+1}}{1-\beta_{n+1}}\left(y_{n+1}-y_{n}\right) \\
& +\left(\frac{\alpha_{n+1}}{1-\beta_{n+1}}-\frac{\alpha_{n}}{1-\beta_{n}}\right)\left(f\left(x_{n}\right)-y_{n}\right) \| \\
& \leq\left|\frac{\alpha_{n+1}}{1-\beta_{n+1}}\right|\left\|f\left(x_{n+1}\right)-f\left(x_{n}\right)\right\|+\left|\frac{\gamma_{n+1}}{1-\beta_{n+1}}\right|\left\|y_{n+1}-y_{n}\right\| \\
& +\left|\left(\frac{\alpha_{n+1}}{1-\beta_{n+1}}-\frac{\alpha_{n}}{1-\beta_{n}}\right)\right|\left\|f\left(x_{n}\right)-y_{n}\right\| \\
& =\left|\frac{\alpha_{n+1}}{1-\beta_{n+1}}\right|\left\|f\left(x_{n+1}\right)-f\left(x_{n}\right)\right\|+\left|1-\frac{\alpha_{n+1}}{1-\beta_{n+1}}\right|\left\|y_{n+1}-y_{n}\right\| \\
& +\left|\left(\frac{\alpha_{n+1}}{1-\beta_{n+1}}-\frac{\alpha_{n}}{1-\beta_{n}}\right)\right|\left\|f\left(x_{n}\right)-y_{n}\right\| \\
& \leq\left|\frac{\alpha_{n+1}}{1-\beta_{n+1}}\right|\left\|f\left(x_{n+1}\right)-f\left(x_{n}\right)\right\|+\left\|y_{n+1}-y_{n}\right\| \\
& +\left|\left(\frac{\alpha_{n+1}}{1-\beta_{n+1}}-\frac{\alpha_{n}}{1-\beta_{n}}\right)\right|\left\|f\left(x_{n}\right)-y_{n}\right\| \\
& \leq\left|\frac{\alpha_{n+1}}{1-\beta_{n+1}}\right|\left\|f\left(x_{n+1}\right)-f\left(x_{n}\right)\right\|+\left|\delta_{n+1}\right|\left\|x_{n+1}-x_{n}\right\|+\left|\delta_{n+1}-\delta_{n}\right|\left(\left\|x_{n}\right\|+\left\|T\left(\mu_{n}\right) x_{n}\right\|\right) \\
& +\left\|T\left(\mu_{n+1}\right) x_{n+1}-T\left(\mu_{n}\right) x_{n+1}\right\|+\left\|x_{n+1}-x_{n}\right\|+c_{n} \\
& +\left|\left(\frac{\alpha_{n+1}}{1-\beta_{n+1}}-\frac{\alpha_{n}}{1-\beta_{n}}\right)\right|\left\|f\left(x_{n}\right)-y_{n}\right\| \text {. }
\end{aligned}
$$

It follows that

$$
\begin{aligned}
\left\|z_{n+1}-z_{n}\right\|-\left\|x_{n+1}-x_{n}\right\| \leq & \left(\frac{\alpha_{n+1}}{1-\beta_{n+1}}+\delta_{n+1}\right)\left\|x_{n+1}-x_{n}\right\| \\
& +\left|\frac{\alpha_{n+1}}{1-\beta_{n+1}}-\frac{\alpha_{n}}{1-\beta_{n}}\right|\left(\left\|f\left(x_{n}\right)\right\|+\left\|y_{n}\right\|\right) \\
& +\left|\delta_{n+1}-\delta_{n}\right|\left(\left\|x_{n}\right\|+\left\|T\left(\mu_{n}\right) x_{n}\right\|\right) \\
& +\left\|T\left(\mu_{n+1}\right) x_{n+1}-T\left(\mu_{n}\right) x_{n+1}\right\|+c_{n} .
\end{aligned}
$$


From (i), (ii), (iv), (3.3) and Lemma 2.3, we have

$$
\limsup _{n \rightarrow \infty}\left(\left\|z_{n+1}-z_{n}\right\|-\left\|x_{n+1}-x_{n}\right\|\right) \leq 0
$$

Applying Lemma 2.7, we obtain $\lim _{n \rightarrow \infty}\left\|z_{n}-x_{n}\right\|=0$ and also

$$
\left\|x_{n+1}-x_{n}\right\|=\left(1-\beta_{n}\right)\left\|z_{n}-x_{n}\right\| \rightarrow 0, \quad \text { as } n \rightarrow \infty .
$$

That is,

$$
\lim _{n \rightarrow \infty}\left\|x_{n+1}-x_{n}\right\|=0
$$

Next, we will show that the set of all limit points of $\left\{x_{n}\right\}$ is a subset of $F(\mathcal{S})$. Note that

$$
\begin{aligned}
\left\|x_{n+1}-x_{n}\right\| & =\left\|\alpha_{n} f\left(x_{n}\right)+\beta_{n} x_{n}+\gamma_{n} y_{n}\right\| \\
& =\left\|\alpha_{n} f\left(x_{n}\right)+\beta_{n} x_{n}+\gamma_{n}\left(\delta_{n} x_{n}+\left(1-\delta_{n}\right) T\left(\mu_{n}\right) x_{n}\right)-x_{n}\right\| \\
& =\left\|\alpha_{n} f\left(x_{n}\right)-\left(1-\beta_{n}\right) x_{n}+\gamma_{n}\left(\delta_{n} x_{n}+\left(1-\delta_{n}\right) T\left(\mu_{n}\right) x_{n}\right)\right\| \\
& =\left\|\alpha_{n} f\left(x_{n}\right)-\left(1-\beta_{n}\right) x_{n}+\gamma_{n} \delta_{n} x_{n}+\gamma_{n} T\left(\mu_{n}\right) x_{n}-\gamma_{n} \delta_{n} T\left(\mu_{n}\right) x_{n}\right\| \\
& =\left\|\alpha_{n} f\left(x_{n}\right)-\left(1-\beta_{n}\right) x_{n}+\gamma_{n} \delta_{n} x_{n}+\left(1-\alpha_{n}-\beta_{n}\right) T\left(\mu_{n}\right) x_{n}-\gamma_{n} \delta_{n} T\left(\mu_{n}\right) x_{n}\right\| \\
& =\left\|\alpha_{n}\left(f\left(x_{n}\right)-T\left(\mu_{n}\right) x_{n}\right)+\left(1-\beta_{n}\right)\left(T\left(\mu_{n}\right) x_{n}-x_{n}\right)+\gamma_{n} \delta_{n}\left(x_{n}-T\left(\mu_{n}\right) x_{n}\right)\right\| \\
& =\left\|\alpha_{n}\left(f\left(x_{n}\right)-T\left(\mu_{n}\right) x_{n}\right)+\left(-1+\beta_{n}+\gamma_{n} \delta_{n}\right)\left(x_{n}-T\left(\mu_{n}\right) x_{n}\right)\right\| \\
& \leq \alpha_{n}\left\|f\left(x_{n}\right)-T\left(\mu_{n}\right) x_{n}\right\|+\left(-1+\beta_{n}+\gamma_{n} \delta_{n}\right)\left\|x_{n}-T\left(\mu_{n}\right) x_{n}\right\| .
\end{aligned}
$$

It follows that

$$
\left\|x_{n}-T\left(\mu_{n}\right) x_{n}\right\| \leq \frac{1}{1-\beta_{n}-\gamma_{n} \delta_{n}}\left(\alpha_{n}\left\|f\left(x_{n}\right)-T\left(\mu_{n}\right) x_{n}\right\|-\left\|x_{n+1}-x_{n}\right\|\right) .
$$

From (i), (ii), (iv) and (3.4), we have

$$
\lim _{n \rightarrow \infty}\left\|x_{n}-T\left(\mu_{n}\right) x_{n}\right\|=0
$$

Let $p$ be a limit point of $\left\{x_{n}\right\}$ and $\left\{x_{n_{k}}\right\}$ be a subsequence of $\left\{x_{n}\right\}$ converging strongly to $p$. From Lemma 2.3, we obtain that

$$
\begin{aligned}
\limsup _{k \rightarrow \infty}\left\|p-T\left(\mu_{n_{k}}\right) p\right\| \leq & \limsup _{k \rightarrow \infty}\left(\left\|p-x_{n_{k}}\right\|+\left\|x_{n_{k}}-T\left(\mu_{n_{k}}\right) x_{n_{k}}\right\|\right. \\
& \left.+\left\|T\left(\mu_{n_{k}}\right) x_{n_{k}}-T\left(\mu_{n_{k}}\right) p\right\|\right) \\
\leq & \limsup _{k \rightarrow \infty}\left(2\left\|p-x_{n_{k}}\right\|+\left\|x_{n_{k}}-T\left(\mu_{n_{k}}\right) x_{n_{k}}\right\|+c_{n_{k}}\right) \\
\leq & 0 .
\end{aligned}
$$

From (3.5), (2.4) and Corollary 2.2, we get $p \in F(\mathcal{S})$. 
We know that there exists a unique sunny nonexpansive retraction $P$ of $C$ onto $F(\mathcal{S})$, and from the Banach contraction mapping principle, we known that $P f$ has a unique fixed point $q$ which by (2.3) is the unique solution of

$$
\langle(f-I) q, J(p-q)\rangle \leq 0, \quad \forall p \in F(\mathcal{S}) .
$$

Let $\left\{x_{n_{k}}\right\}$ be a subsequence of $\left\{x_{n}\right\}$ converging to $p \in C$. From the smoothness of $E$ and (3.6), we have that

$$
\begin{aligned}
\limsup _{n \rightarrow \infty}\left\langle(f-I) q, J\left(x_{n}-q\right)\right\rangle & =\limsup _{k \rightarrow \infty}\left\langle(f-I) q, J\left(x_{n_{k}}-q\right)\right\rangle \\
& =\langle(f-I) q, J(p-q)\rangle \leq 0 .
\end{aligned}
$$

Finally, we show that the sequence $\left\{x_{n}\right\}$ converges strongly to $q=P f q$. Now, we have

$$
\begin{aligned}
\left\|y_{n}-q\right\| & =\left\|\delta_{n} x_{n}+\left(1-\delta_{n}\right) T\left(\mu_{n}\right) x_{n}-q\right\| \\
& =\left\|\left(1-\delta_{n}\right)\left(T\left(\mu_{n}\right) x_{n}-q\right)+\delta_{n}\left(x_{n}-q\right)\right\| \\
& \leq\left(1-\delta_{n}\right)\left\|T\left(\mu_{n}\right) x_{n}-q\right\|+\delta_{n}\left\|x_{n}-q\right\| \\
& \leq\left(1-\delta_{n}\right)\left\|x_{n}-q\right\|+c_{n}+\delta_{n}\left\|x_{n}-q\right\| \\
& =\left\|x_{n}-q\right\|+c_{n} .
\end{aligned}
$$

From Lemma 2.6, (3.8) and (2.2), we have

$$
\begin{aligned}
\left\|x_{n+1}-q\right\|^{2}= & \left\|\alpha_{n} f\left(x_{n}\right)+\beta_{n} x_{n}+\gamma_{n} y_{n}-q\right\|^{2} \\
= & \left\|\left(\gamma_{n}\left(y_{n}-q\right)+\beta_{n}\left(x_{n}-q\right)\right)+\alpha_{n}\left(f\left(x_{n}\right)-q\right)\right\|^{2} \\
\leq & \left\|\gamma_{n}\left(y_{n}-q\right)+\beta_{n}\left(x_{n}-q\right)\right\|^{2}+2 \alpha_{n}\left\langle f\left(x_{n}\right)-q, J\left(x_{n+1}-q\right)\right\rangle \\
= & \left\|\left(1-\beta_{n}\right) \frac{\gamma_{n}}{1-\beta_{n}}\left(y_{n}-q\right)+\beta_{n}\left(\frac{1-\beta_{n}}{1-\beta_{n}}\right)\left(x_{n}-q\right)\right\|^{2} \\
& +2 \alpha_{n}\left\langle f\left(x_{n}\right)-f(q), J\left(x_{n+1}-q\right)\right\rangle+2 \alpha_{n}\left\langle f(q)-q, J\left(x_{n+1}-q\right)\right\rangle \\
\leq & \left(1-\beta_{n}\right)\left\|\frac{\gamma_{n}}{1-\beta_{n}}\left(y_{n}-q\right)\right\|^{2}+\beta_{n}\left\|x_{n}-q\right\|^{2} \\
& +2 r \alpha_{n}\left\|x_{n}-q\right\|\left\|x_{n+1}-q\right\|+2 \alpha_{n}\left\langle f(q)-q, J\left(x_{n+1}-q\right)\right\rangle \\
\leq & \frac{\gamma_{n}^{2}}{1-\beta_{n}}\left\|y_{n}-q\right\|^{2}+\beta_{n}\left\|x_{n}-q\right\|^{2} \\
& +r \alpha_{n}\left(\left\|x_{n}-q\right\|^{2}+\left\|x_{n+1}-q\right\|^{2}\right)+2 \alpha_{n}\left\langle f(q)-q, J\left(x_{n+1}-q\right)\right\rangle \\
\leq & \frac{\gamma_{n}^{2}}{1-\beta_{n}}\left\|x_{n}-q\right\|^{2}+\frac{\gamma_{n}^{2} c_{n}}{1-\beta_{n}}+\beta_{n}\left\|x_{n}-q\right\|^{2} \\
& +r \alpha_{n}\left\|x_{n}-q\right\|^{2}+r \alpha_{n}\left\|x_{n+1}-q\right\|^{2}+2 \alpha_{n}\left\langle f(q)-q, J\left(x_{n+1}-q\right)\right\rangle \\
= & \left(\frac{\gamma_{n}^{2}}{1-\beta_{n}}+\beta_{n}+r \alpha_{n}\right)\left\|x_{n}-q\right\|^{2}+\frac{\gamma_{n}^{2} c_{n}}{1-\beta_{n}} \\
& +r \alpha_{n}\left\|x_{n+1}-q\right\|^{2}+2 \alpha_{n}\left\langle f(q)-q, J\left(x_{n+1}-q\right)\right\rangle
\end{aligned}
$$




$$
\begin{aligned}
= & \left(\frac{\left(\left(1-\beta_{n}\right)-\alpha_{n}\right)^{2}}{1-\beta_{n}}+\beta_{n}+r \alpha_{n}\right)\left\|x_{n}-q\right\|^{2}+\frac{\gamma_{n}^{2} c_{n}}{1-\beta_{n}} \\
& +r \alpha_{n}\left\|x_{n+1}-q\right\|^{2}+2 \alpha_{n}\left\langle f(q)-q, J\left(x_{n+1}-q\right)\right\rangle \\
= & \left(\frac{\left(1-\beta_{n}\right)^{2}-2\left(1-\beta_{n}\right) \alpha_{n}+\alpha_{n}^{2}}{1-\beta_{n}}+\beta_{n}+r \alpha_{n}\right)\left\|x_{n}-q\right\|^{2} \\
& +\frac{\gamma_{n}^{2} c_{n}}{1-\beta_{n}}+r \alpha_{n}\left\|x_{n+1}-q\right\|^{2}+2 \alpha_{n}\left\langle f(q)-q, J\left(x_{n+1}-q\right)\right\rangle \\
= & \left(1-\beta_{n}-2 \alpha_{n}+\frac{\alpha_{n}^{2}}{1-\beta_{n}}+\beta_{n}+r \alpha_{n}\right)\left\|x_{n}-q\right\|^{2}+\frac{\gamma_{n}^{2} c_{n}}{1-\beta_{n}} \\
& +r \alpha_{n}\left\|x_{n+1}-q\right\|^{2}+2 \alpha_{n}\left\langle f(q)-q, J\left(x_{n+1}-q\right)\right\rangle \\
= & \left(\left(1-r \alpha_{n}\right)+\left(2 r \alpha_{n}-2 \alpha_{n}\right)+\frac{\alpha_{n}^{2}}{1-\beta_{n}}\right)\left\|x_{n}-q\right\|^{2}+\frac{\gamma_{n}^{2} c_{n}}{1-\beta_{n}} \\
& +r \alpha_{n}\left\|x_{n+1}-q\right\|^{2}+2 \alpha_{n}\left\langle f(q)-q, J\left(x_{n+1}-q\right)\right\rangle .
\end{aligned}
$$

It follows that

$$
\begin{aligned}
\left\|x_{n+1}-q\right\|^{2} \leq & \left(1-\frac{2 \alpha_{n}(1-r)}{1-r \alpha_{n}}+\frac{\alpha_{n}^{2}}{\left(1-r \alpha_{n}\right)\left(1-\beta_{n}\right)}\right)\left\|x_{n}-q\right\|^{2}+\frac{\gamma_{n}^{2} c_{n}}{\left(1-r \alpha_{n}\right)\left(1-\beta_{n}\right)} \\
& +\frac{2 \alpha_{n}}{1-r \alpha_{n}}\left\langle f(q)-q, J\left(x_{n+1}-q\right)\right\rangle \\
\leq & \left(1-\frac{2 \alpha_{n}(1-r)}{1-r \alpha_{n}}\right)\left\|x_{n}-q\right\|^{2}+\frac{\alpha_{n}}{1-r \alpha_{n}}\left(\frac{\alpha_{n}}{1-\beta_{n}}\left\|x_{n}-q\right\|^{2}\right. \\
& \left.+\frac{\gamma_{n}^{2} c_{n}}{\alpha_{n}\left(1-\beta_{n}\right)}+2\left\langle f(q)-q, J\left(x_{n+1}-q\right)\right\rangle\right) \\
:= & \left(1-\sigma_{n}\right)\left\|x_{n}-q\right\|^{2}+\rho_{n},
\end{aligned}
$$

where $\sigma_{n}:=\frac{2 \alpha_{n}(1-r)}{1-r \alpha_{n}}$ and $\rho_{n}:=\frac{\alpha_{n}}{1-r \alpha_{n}}\left(\frac{\alpha_{n}}{1-\beta_{n}}\left\|x_{n}-q\right\|^{2}+\frac{\gamma_{n}^{2} c_{n}}{\alpha_{n}\left(1-\beta_{n}\right)}+2\left\langle f(q)-q, J\left(x_{n+1}-q\right)\right\rangle\right)$. Now, from (i), (iii), (iv), (3.7) and Lemma 2.8, $\left\|x_{n}-q\right\| \rightarrow 0$ as $n \rightarrow \infty$. This proof is completed.

Corollary 3.2 Let $C$ be a nonempty, compact and convex subset of a smooth Banach space E. Let $S$ be a left reversible semigroup and $\mathcal{S}=\left\{T_{s}: s \in S\right\}$ be a representation of $S$ as Lipschitzian mappings from $C$ into itself, with the uniform Lipschitzian condition $\lim _{s} k_{s} \leq 1$, and $f$ be an $\alpha$-contraction of $C$ into itself. Let $X$ be a left invariant $\mathcal{S}$-stable subspace of $l^{\infty}(S)$ containing $1,\left\{\mu_{n}\right\}$ be a strongly left regular sequence of means on $X$ such that $\lim _{n \rightarrow \infty}\left\|\mu_{n+1}-\mu_{n}\right\|=0$ and $\left\{c_{n}\right\}$ be the sequence defined by (2.4) with $\limsup _{n \rightarrow \infty} c_{n} \leq 0$. Suppose the sequences $\left\{\alpha_{n}\right\},\left\{\beta_{n}\right\},\left\{\gamma_{n}\right\}$ and $\left\{\delta_{n}\right\}$ in $(0,1)$ satisfy $\alpha_{n}+\beta_{n}+\gamma_{n}=1, n \geq 1$. The following conditions are satisfied:

(i) $\lim _{n \rightarrow \infty} \alpha_{n}=0$ and $\sum_{n=0}^{\infty} \alpha_{n}=\infty$;

(ii) $\lim _{n \rightarrow \infty} \delta_{n}=0$;

(iii) $\lim \sup _{n \rightarrow \infty} \frac{c_{n}}{\alpha_{n}} \leq 0$;

(iv) $0<\liminf _{n \rightarrow \infty} \beta_{n} \leq \limsup _{n \rightarrow \infty} \beta_{n}<1$.

For arbitrary given $x_{1} \in C$, generate a sequence $\left\{x_{n}\right\}$ by

$$
\left\{\begin{array}{l}
y_{n}=\delta_{n} x_{n}+\left(1-\delta_{n}\right) T\left(\mu_{n}\right) x_{n}, \\
x_{n+1}=\alpha_{n} f\left(x_{n}\right)+\beta_{n} x_{n}+\gamma_{n} y_{n} .
\end{array}\right.
$$


Then $\left\{x_{n}\right\}$ converges strongly to $q \in F(\mathcal{S})$, which is the unique solution of the variational inequality

$$
\langle(f-I) q, J(p-q)\rangle \leq 0, \quad \forall p \in F(\mathcal{S})
$$

Equivalently, we have $q=P f q$, where $P$ is the unique sunny nonexpansive retraction of $C$ onto $F(\mathcal{S})$.

Corollary 3.3 Let $C$ be a nonempty, compact and convex subset of a smooth Banach space E. Let $S$ be a left reversible semigroup and $\mathcal{S}=\left\{T_{s}: s \in S\right\}$ be a representation of $S$ as Lipschitzian mappings from $C$ into itself, with the uniform Lipschitzian condition $\lim _{s} k_{s} \leq 1$, and $f$ be a Meir-Keeler contraction of $C$ into itself. Let $X$ be a left invariant $\mathcal{S}$-stable subspace of $l^{\infty}(S)$ containing $1,\left\{\mu_{n}\right\}$ be a strongly left regular sequence of means on $X$ such that $\lim _{n \rightarrow \infty}\left\|\mu_{n+1}-\mu_{n}\right\|=0$ and $\left\{c_{n}\right\}$ be the sequence defined by (2.4) with $\limsup _{n \rightarrow \infty} c_{n} \leq 0$. Suppose the sequences $\left\{\alpha_{n}\right\},\left\{\beta_{n}\right\}$ and $\left\{\gamma_{n}\right\}$ in $(0,1)$ satisfy $\alpha_{n}+\beta_{n}+\gamma_{n}=1$, $n \geq 1$. The following conditions are satisfied:

(i) $\lim _{n \rightarrow \infty} \alpha_{n}=0$ and $\sum_{n=0}^{\infty} \alpha_{n}=\infty$;

(ii) $\lim \sup _{n \rightarrow \infty} \frac{c_{n}}{\alpha_{n}} \leq 0$;

(iii) $0<\liminf _{n \rightarrow \infty} \beta_{n} \leq \limsup _{n \rightarrow \infty} \beta_{n}<1$

For arbitrary $x_{1} \in C$, generate a sequence $\left\{x_{n}\right\}$ by

$$
x_{n+1}=\alpha_{n} f\left(x_{n}\right)+\beta_{n} x_{n}+\gamma_{n} T\left(\mu_{n}\right) x_{n} .
$$

Then $\left\{x_{n}\right\}$ converges strongly to $q \in F(\mathcal{S})$, which is the unique solution of the variational inequality

$$
\langle(f-I) q, J(p-q)\rangle \leq 0, \quad \forall p \in F(\mathcal{S}) .
$$

Equivalently, we have $q=P f q$, where $P$ is the unique sunny nonexpansive retraction of $C$ onto $F(\mathcal{S})$.

Corollary 3.4 Let $C$ be a nonempty, compact and convex subset of a smooth Banach space $E$. Let $S$ be a left reversible semigroup and $\mathcal{S}=\left\{T_{s}: s \in S\right\}$ be a representation of $S$ as Lipschitzian mappings from $C$ into itself, with the uniform Lipschitzian condition $\lim _{s} k_{s} \leq 1$, and $f$ be an $\alpha$-contraction of $C$ into itself. Let $X$ be a left invariant $\mathcal{S}$-stable subspace of $l^{\infty}(S)$ containing $1,\left\{\mu_{n}\right\}$ be a strongly left regular sequence of means on $X$ such that $\lim _{n \rightarrow \infty}\left\|\mu_{n+1}-\mu_{n}\right\|=0$ and $\left\{c_{n}\right\}$ be the sequence defined by (2.4) with $\lim _{\sup _{n \rightarrow \infty}} c_{n} \leq 0$. Suppose the sequences $\left\{\alpha_{n}\right\},\left\{\beta_{n}\right\}$ and $\left\{\gamma_{n}\right\}$ in $(0,1)$ satisfy $\alpha_{n}+\beta_{n}+\gamma_{n}=1, n \geq 1$. The following conditions are satisfied:

(i) $\lim _{n \rightarrow \infty} \alpha_{n}=0$ and $\sum_{n=0}^{\infty} \alpha_{n}=\infty$;

(ii) $\limsup _{n \rightarrow \infty} \frac{c_{n}}{\alpha_{n}} \leq 0$;

(iii) $0<\liminf _{n \rightarrow \infty} \beta_{n} \leq \limsup _{n \rightarrow \infty} \beta_{n}<1$.

For arbitrary $x_{1} \in C$, generate a sequence $\left\{x_{n}\right\}$ by

$$
x_{n+1}=\alpha_{n} f\left(x_{n}\right)+\beta_{n} x_{n}+\gamma_{n} T\left(\mu_{n}\right) x_{n} .
$$


Then $\left\{x_{n}\right\}$ converges strongly to $q \in F(\mathcal{S})$, which is the unique solution of the variational inequality

$$
\langle(f-I) q, J(p-q)\rangle \leq 0, \quad \forall p \in F(\mathcal{S}) .
$$

Equivalently, we have $q=P f q$, where $P$ is the unique sunny nonexpansive retraction of $C$ onto $F(\mathcal{S})$.

Corollary 3.5 Let $C$ be a nonempty, compact and convex subset of a smooth Banach space $E$. Let $S$ be a left reversible semigroup and $\mathcal{S}=\left\{T_{s}: s \in S\right\}$ be a representation of $S$ as nonexpansive mappings from $C$ into itself and $f$ be an $\alpha$-contraction of $C$ into itself. Let $X$ be a left invariant $\mathcal{S}$-stable subspace of $l^{\infty}(S)$ containing $1,\left\{\mu_{n}\right\}$ be a strongly left regular sequence of means on $X$ such that $\lim _{n \rightarrow \infty}\left\|\mu_{n+1}-\mu_{n}\right\|=0$ and $\left\{c_{n}\right\}$ be the sequence defined by (2.4) with $\limsup _{n \rightarrow \infty} c_{n} \leq 0$. Suppose the sequences $\left\{\alpha_{n}\right\},\left\{\beta_{n}\right\},\left\{\gamma_{n}\right\}$ and $\left\{\delta_{n}\right\}$ in $(0,1)$ satisfy $\alpha_{n}+\beta_{n}+\gamma_{n}=1, n \geq 1$. The following conditions are satisfied:

(i) $\lim _{n \rightarrow \infty} \alpha_{n}=0$ and $\sum_{n=0}^{\infty} \alpha_{n}=\infty$;

(ii) $\lim _{n \rightarrow \infty} \delta_{n}=0$;

(iii) $\lim \sup _{n \rightarrow \infty} \frac{c_{n}}{\alpha_{n}} \leq 0$;

(iv) $0<\liminf _{n \rightarrow \infty} \beta_{n} \leq \limsup _{n \rightarrow \infty} \beta_{n}<1$.

For arbitrary $x_{1} \in C$, generate a sequences $\left\{x_{n}\right\}$ by

$$
\left\{\begin{array}{l}
y_{n}=\delta_{n} x_{n}+\left(1-\delta_{n}\right) T\left(\mu_{n}\right) x_{n}, \\
x_{n+1}=\alpha_{n} f\left(x_{n}\right)+\beta_{n} x_{n}+\gamma_{n} y_{n} .
\end{array}\right.
$$

Then $\left\{x_{n}\right\}$ converges strongly to $q \in F(\mathcal{S})$, which is the unique solution of the variational inequality

$$
\langle(f-I) q, J(p-q)\rangle \leq 0, \quad \forall p \in F(\mathcal{S}) .
$$

Equivalently, we have $q=P f q$, where $P$ is the unique sunny nonexpansive retraction of $C$ onto $F(\mathcal{S})$.

\section{Competing interests}

The authors declare that they have no competing interests.

\section{Authors' contributions}

All authors contributed equally and significantly in writing this article. All authors read and approved the final manuscript.

\section{Acknowledgements}

The authors would like to express their thanks to referees for the careful reading of the paper and for the suggestions which improved the quality of this work.

Received: 22 July 2012 Accepted: 15 November 2012 Published: 3 December 2012

\section{References}

1. Mann, WR: Mean value methods in iteration. Proc. Am. Math. Soc. 4, 506-510 (1953)

2. Reich, S, Zaslavski, AJ: Convergence of Krasnoselskii-Mann iterations of nonexpansive operators. Math. Comput. Model. 32, 1423-1431 (2000)

3. Halpern, B: Fixed points of nonexpanding maps. Bull. Am. Math. Soc. 73, 957-961 (1967)

4. Xu, HK: Viscosity approximation methods for nonexpansive mappings. J. Math. Anal. Appl. 298, 279-291 (2004)

5. Lau, AT, Miyake, H, Takahashi, W: Approximation of fixed points for amenable semigroups of nonexpansive mappings in Banach spaces. Nonlinear Anal. 67(4), 1211-1225 (2007) 
6. Zhang, S-S, Yang, L, Liu, J-A: Strong convergence theorems for nonexpansive semigroups in Banach spaces. Appl. Math. Mech. 28(10), 1287-1297 (2007)

7. Saeidi, S: Approximating common fixed points of Lipschitzian semigroup in smooth Banach spaces. Fixed Point Theory Appl. 2008, Article ID 363257 (2008)

8. Kirk, WA, Sims, B: Handbook of Metric Fixed Point Theory. Kluwer Academic, Dordrecht (2001)

9. Takahashi, W: Nonlinear Functional Analysis. Yokohama Publishers, Yokohama (2000)

10. Hindman, N, Strauss, D: Density and invariant means in left amenable semigroups. Topol. Appl. 156, 2614-2628 (2009)

11. Holmes, RD, Lau, AT: Non-expansive actions of topological semigroups and fixed points. J. Lond. Math. Soc. 5, 330-336 (1972)

12. Hirano, N, Kido, K, Takahashi, W: Nonexpansive retractions and nonlinear ergodic theorems in Banach spaces. Nonlinear Anal. 12(11), 1263-1281 (1988)

13. Saeidi, S: Existence of ergodic retractions for semigroups in Banach spaces. Nonlinear Anal. 69(10), 3417-3422 (2008)

14. Takahashi, W: A nonlinear ergodic theorem for an amenable semigroup of nonexpansive mappings in a Hilbert space. Proc. Am. Math. Soc. 81(2), 253-256 (1981)

15. Reich, S: Asymptotic behavior of contractions in Banach spaces. J. Math. Anal. Appl. 44(1), 57-70 (1973)

16. Saeidi, S: Strong convergence of Browder's type iterations for left amenable semigroups of Lipschitzian mappings in Banach spaces. J. Fixed Point Theory Appl. 5, 93-103 (2009)

17. Suzuki, T: Strong convergence of Krasnoselskii and Mann's type sequences for one-parameter nonexpansive semigroups without Bochner integrals. J. Math. Anal. Appl. 305(1), 227-239 (2005)

18. Lim, TC: On characterizations of Meir-Keeler contractive maps. Nonlinear Anal. 46(1), 113-120 (2001)

19. Petrusel, A, Yao, JC: Viscosity approximation to common fixed points of families of nonexpansive mappings with generalized contractions mappings. Nonlinear Anal. 69(4), 1100-1111 (2008)

doi:10.1186/1029-242X-2012-279

Cite this article as: Saewan and Kumam: Explicit iterations for Lipschitzian semigroups with the Meir-Keeler type contraction in Banach spaces. Journal of Inequalities and Applications 2012 2012:279.

\section{Submit your manuscript to a SpringerOpen ${ }^{\ominus}$ journal and benefit from:}

- Convenient online submission

- Rigorous peer review

- Immediate publication on acceptance

- Open access: articles freely available online

- High visibility within the field

- Retaining the copyright to your article 